\title{
Reducción de la infección perineal tras la resección abdomino-perineal por cáncer de recto mediante la malla de colágeno impregnada con gentamicina
}

\author{
Cristina Isabel Martínez ${ }^{1}$, Luis Jorge Lombana ${ }^{1}$, Andrés Monroy², David Baquero²
}

Palabras clave: neoplasias del recto; prótesis e implantes; colágeno; antibacterianos; complicaciones posoperatorias; infección de la herida quirúrgica.

\section{Resumen}

Introducción. La resección abdomino-perineal en pacientes con cáncer de recto sometidos a radioterapia preoperatoria tiene como complicación frecuente la infección perineal del sitio operatorio, que se manifiesta desde celulitis hasta colecciones perineales y dehiscencia de la herida lo que aumenta de manera significativa el tiempo de la hospitalizacion.

El reducir la incidencia de la infección del sitio operatorio contribuye a disminuir la morbimortalidad perioperatoria. El uso local de antibióticos en la herida quirúrgica se ha reportado en la literatura como un factor protector con resultados promisorios para evitar esta complicación.

Médico coloproctólogo; jefe, Departamento de Coloproctología, Hospital San Ignacio; fellow de Coloproctología, Universidad del Rosario, Bogotá, D.C., Colombia

2 Médico coloproctólogo, Hospital Universitario Mayor Mederi; docente de Coloproctología, Universidad del Rosario; fellow de Coloproctología, Universidad del Rosario, Bogotá, D.C., Colombia

Fecha de recibido: 17 de mayo de 2017

Fecha de aprobación: 8 de septiembre de 2017

Citar como: Martínez CI, Lombana LJ, Monroy A, Baquero D. Reducción de la infección perineal tras la resección abdomino-perineal por cáncer de recto mediante la malla de colágeno impregnada con gentamicina. Rev Colomb Cir. 2017;32:277-82.
Métodos. Se presenta una serie de casos en la que se analizan los resultados de 12 pacientes con diagnóstico de cáncer de recto, sometidos a resección abdomino-perineal más colocación de malla de colágeno impregnada con gentamicina en el cierre perineal. El estudio incluyó pacientes atendidos en el Servicio de Coloproctología del Hospital Universitario San Ignacio.

Su principal objetivo fue describir la experiencia institucional con este tipo de mallas como medida preventiva para disminuir la infección perineal en estos pacientes.

Resultados. La incidencia de infección fue de $41 \%$ con la utilización de la malla de gentamicina. Nueve de los 12 pacientes habian recibido neoadyuvancia con quimiorradioterapia y tres fueron intervenidos quirúrgicamente sin neoadyuvancia previa por presentar tumores $T_{1} / T_{2}$. Se presentó infección del sitio operatorio en cinco pacientes, en tres fue de tipo superficial y, en dos, de órgano y espacio y requirió manejo quirúrgico. No se demostraron efectos secundarios de la malla con gentamicina.

Conclusiones. Los resultados muestran una tendencia positiva de la efectividad de las mallas con gentamicina para disminuir la aparición de infección del sitio operatorio. En comparación con los pacientes intervenidos sin neoadyuvancia, los que reciben neoadyuvancia tienen mayor riesgo de infección del sitio operatorio. La implementación de las mallas con gentamicina no supone una modificación mayor de la técnica quirúr- 
gica ni aumenta la morbilidad del procedimiento. Si bien estas observaciones son indicios de que el uso de mallas con antibiótico tópico tiene un impacto positivo en la reducción de infección del sitio operatorio, se requieren estudios futuros para obtener resultados estadísticamente significativos.

\section{Introducción}

Las infecciones del sitio operatorio son un problema frecuente asociado a cualquier tipo de procedimiento quirúrgico y generan un aumento de la morbimortalidad perioperatoria, del tiempo de hospitalización y de los costos por la necesidad de tratamientos antimicrobianos y procedimientos quirúrgicos adicionales ${ }^{1,2}$.

Se han identificado numerosos factores de riesgo para desarrollar la infección del sitio operatorio, incluyendo los derivados del procedimiento quirúrgico, la técnica y el tipo de cirugía y, por otro lado, los relacionados con el paciente, como obesidad, diabetes, compromiso inmunológico, enfermedades concomitantes, cirugía de urgencia y pérdida sanguínea ${ }^{3,4}$.

El U.S National Research Council Group desarrolló un sistema de clasificación para heridas quirúrgicas, basado en el grado de contaminación bacteriana, que las agrupa en cuatro diferentes grupos: limpia, limpia-contaminada, contaminada y sucia. Esto permite establecer el riesgo de desarrollar infección del sitio operatorio y la necesidad de instaurar profilaxis antimicrobiana ${ }^{5}$. Una buena técnica quirúrgica, adecuadas asepsia y antisepsia, y la profilaxis antibiótica, son esenciales para la prevención de esta complicación.

Hay datos que demuestran que la profilaxis con antibióticos sistémicos disminuye la incidencia de infección del sitio operatorio, pero muchas veces hay factores asociados con el procedimiento que no permiten una concentración adecuada del antibiótico en el sitio quirúrgico ${ }^{6}$. En el caso de las cirugías de recto, y especialmente en pacientes que han sido previamente irradiados como parte de su tratamiento neoadyuvante, se generan condiciones locales que son adversas para la penetración del antibiótico sistémico en estos tejidos. La fibrosis inducida por la radiación, sumada a la disección rigurosa del mesenterio del recto, produce deficiente perfusión sanguínea, mala oxigenación de los tejidos de esta zona y tendencia a la exudación de capilares linfáticos. El resultado es la formación de una barrera de fibrosis y granulación al paso de los antibióticos, lo que conlleva un mayor riesgo de infección, de dehiscencia de la herida y de cicatrización retardada ${ }^{7}$.

Existen reportes en la literatura sobre el uso de mallas de colágeno impregnadas con gentamicina, en los cuales se ha demostrado una disminución de la tasa de infección del periné ${ }^{8-10}$. Estas mallas de colágeno se absorben con la actividad normal de las colagenasas de la herida, en el proceso de cicatrización, mientras liberan localmente el antibiótico. Además, aumentan las concentraciones de antibiótico local, por encima de la concentración inhibitoria mínima (CIM), al mismo tiempo que generan toxicidad sistémica mínima porque tienen muy poca absorción en los tejidos y generan bajos niveles plasmáticos ${ }^{11-13}$.

El objetivo principal del estudio fue describir la experiencia en el Servicio de Coloproctología del Hospital San Ignacio con la utilización de una malla de colágeno impregnada con gentamicina como medida preventiva para disminuir la incidencia de infección perineal en pacientes sometidos a resección abdomino-perineal por cáncer de recto.

\section{Materiales y métodos}

\section{Diseño del estudio}

Se llevó a cabo un estudio observacional descriptivo de tipo reporte de serie de casos, en el que se analizan los resultados de 12 pacientes con diagnóstico de cáncer de recto, sometidos a resección abdomino-perineal más colocación de malla de colágeno impregnada con gentamicina, en el Servicio de Coloproctología del Hospital Universitario San Ignacio, previo consentimiento informado.

Los pacientes incluidos en el estudio fueron sometidos al protocolo convencional, sin ningún cambio en la conducta terapéutica ni variaciones en la técnica quirúrgica $\mathrm{y}$, antes del cierre de la herida perineal, se les colocó una malla de colágeno impregnada con gentamicina. Se hizo seguimiento durante un periodo de cuatro semanas para detectar la aparición de infección del sitio operatorio, según los criterios diagnósticos de los Centers for Disease Control and Prevention (CDC). 
Los datos se recolectaron de forma retrospectiva a partir de la revisión de la historia clínica electrónica de los pacientes seleccionados. Los datos obtenidos se organizaron y archivaron en formato de Excel en las tablas de recolección de datos.

\section{Población de estudio}

Se incluyeron 12 pacientes con diagnóstico de cáncer de recto que fueron sometidos a resección abdóminoperineal en el Servicio de Coloproctología del Hospital Universitario San Ignacio.

Los criterios de inclusión fueron hombres y mujeres mayores de 18 años, con antecedentes de tratamiento neoadyuvantes o sin ellos, sometidos a resección abdómino-perineal por cáncer de recto.

Los criterios de exclusión fueron cambios en la técnica quirúrgica, hallazgo de lesiones irresecables y perforación del recto durante la cirugía.

Las variables que se tuvieron en cuenta en este estudio fueron: edad, sexo, clasificación TNM y antecedentes de tratamiento neoadyuvante, localización del tumor en el recto superior, medio o inferior, y tiempo quirúrgico medido en minutos.

\section{Técnica quirúrgica}

Durante el procedimiento quirúrgico, después de completar la resección perineal, se cortó la malla de colágeno de $5 \times 5 \mathrm{~cm}$ en dos fragmentos, los cuales se introdujeron en la herida perineal a nivel de los músculos elevadores (plano más profundo) y en el tejido celular subcutáneo inmediatamente antes de la piel. La piel se afrontó con puntos separados de material absorbible.

\section{Consideraciones éticas}

Este es un estudio sin riesgo, según la Resolución 008430 de 1993 del Ministerio de Salud de Colombia, Artículo 11. Se respetaron los principios éticos para investigación en humanos enunciados en la Declaración de Helsinki, revisada en octubre de 2000 en Escocia por la Asamblea Médica Mundial, y los de la Resolución del Ministerio de Salud 008430 de 1993.

La confidencialidad de los datos obtenidos se garantizó utilizando el número de la historia clínica como identificación. Se limitó el acceso de los instrumentos de investigación únicamente a los investigadores principales (Resolución 008430 de 1993 del Ministerio de Salud, artículo 8).

\section{Resultados}

Se presentó infección del sitio operatorio en 5 de los 12 pacientes, lo que equivale a $41 \%$ de la muestra. De estos cinco pacientes, en tres, la infección del sitio operatorio fue superficial y fueron tratados con curaciones ambulatorias, y en dos, la infección fue de órgano y espacio, por lo cual fueron intervenidos quirúrgicamente, con lavado y drenaje de la colección. No se demostraron efectos secundarios asociados con la malla impregnada con gentamicina. Los datos obtenidos se clasificaron en categorías para establecer la relación de la infección del sitio operatorio con las variables descritas, y se mencionan a continuación.

\section{Edad}

La mayor frecuencia de infección del sitio operatorio se presentó en pacientes mayores de 60 años: cinco de las seis infecciones perineales se produjeron en este grupo etario.

\section{Sexo}

Nueve pacientes eran de sexo masculino y tres de sexo femenino. Se presentó infección del sitio operatorio en cuatro de los hombres y en una de las mujeres (tabla 1).

\section{Antecedente de neoadyuvancia y clasificación TNM}

En siete de los pacientes la clasificación de los tumores fue $\mathrm{T}_{3} \mathrm{y}$, de estos, cuatro presentaron infección del sitio operatorio, lo cual corresponde a $80 \%$ de aquellos con

TABLA 1.

Características demográficas de la población de estudio $(N=12)$

Tamaño de la muestra

\begin{tabular}{lcc}
\hline \multirow{2}{*}{ Sexo } & Masculino & 9 \\
\cline { 2 - 3 } & Femenino & 3 \\
\hline \multirow{2}{*}{ Edad (años) } & $>60$ & 7 \\
\cline { 2 - 3 } & $40-60$ & 4 \\
\cline { 2 - 3 } & $<40$ & 1 \\
\hline
\end{tabular}


dicha infección. Según el compromiso ganglionar, hubo infección del sitio operatorio en tres de los seis casos con tumores $\mathrm{N}_{0}$, y en dos de los cinco con tumores $\mathrm{N}_{1}$; no se presentó en el paciente con tumor $\mathrm{N}_{2}$ (tabla 2).

Por otro lado, todos los pacientes con tumores $\mathrm{T}_{3} \mathrm{y}$ $\mathrm{T}_{4}$ habían recibido tratamiento neoadyuvante con quimiorradioterapia (9 pacientes). Los tres con tumores $\mathrm{T}_{1} \mathrm{y} \mathrm{T}_{2}$ fueron sometidos a cirugía sin haber recibido neoadyuvancia y en ninguno de ellos se diagnosticó infección del sitio operatorio (tabla 2). Todos los cinco pacientes con infección del sitio operatorio habían recibido quimiorradioterapia.

\section{Localización del tumor}

Del total de la muestra, un paciente tenía cáncer de recto medio y no presentó infección del sitio operatorio, y once pacientes tenían cáncer de recto inferior. Los cinco que presentaron infección del sitio operatorio pertenecían a este último grupo con afección del recto inferior.

\section{Tiempo quirúrgico}

El promedio del tiempo quirúrgico fue de 170 minutos, con un rango entre 150 y 240. El tiempo quirúrgico en los casos de infección del sitio operatorio fue: 150 minutos en dos pacientes, 180 minutos en dos y 240 minutos en uno.

\section{Discusión}

El cáncer colorrectal es más común en los países industrializados; en Estados Unidos ocupa el tercer lugar entre los hombres, después del cáncer de próstata y de pulmón, y entre las mujeres, sigue a los de mama y de pulmón.

El tratamiento del adenocarcinoma rectal ha cambiado durante las últimas décadas, con avances en la técnica quirúrgica, la radioterapia y la quimioterapia, lográndose menos recurrencias locales y regionales y mejores tasas de supervivencia. Pese al entendimiento de la anatomía del aparato esfinteriano y el progreso en el desarrollo de cirugías preservadoras de esfínteres, quedan muchos casos en los cuales la localización del tumor exige resecciones abdomino-perineales para obtener una buena erradicación, que demandan una disección completa del piso pélvico y resección del ano con el aparato esfinteriano ${ }^{14}$.
Todas las heridas quirúrgicas tienen el riesgo potencial de estar contaminadas por bacterias, en especial, aquellas en las que el órgano implicado es el colon, por su gran cantidad de población bacteriana. A pesar de esto, solo en algunas heridas se desarrolla infección clínica que clásicamente se manifiesta como mínimo con uno de los siguientes hallazgos: drenaje purulento con confirmación microbiológica de la infección superficial o sin ella, aislamiento de microorganismos en fluido o tejido obtenidos de la herida, o uno de los siguientes hallazgos como dolor, inflamación, eritema, calor local o diagnóstico hecho por el cirujano ${ }^{15,16}$.

Debido a la gran variedad de conceptos desarrollados a lo largo del tiempo, en 1992 se cambió el término de infección de la herida por infección del sitio operatorio. A finales de los años 90, los Centers for Disease Control and Prevention (CDC) de Estados Unidos clasificaron la infección del sitio operatorio como superficial, profunda o de órgano y espacio. La superficial involucra solo piel y tejido celular subcutáneo; en la profunda, hay compromiso de los tejidos blandos más profundos, como músculo y fascia; y, finalmente, la de órgano y espacio implica la afección de zona anatómica diferente a las capas de

TABLA 2.

Clasificación TNM de los pacientes incluidos en el estudio

\begin{tabular}{lcc}
\hline Clasificación T & Número de pacientes & ISO \\
\hline $\mathrm{T}_{1}$ & 2 & 0 \\
$\mathrm{~T}_{2}$ & 1 & 0 \\
$\mathrm{~T}_{3}$ & 7 & 4 \\
$\mathrm{~T}_{4}$ & 2 & 1 \\
Clasificación N & & \\
$\mathrm{N}_{0}$ & 6 & 3 \\
$\mathrm{~N}_{1}$ & 5 & 2 \\
$\mathrm{~N}_{2}$ & 1 & 0 \\
Clasificación M & & \\
$\mathrm{M}_{0}$ & 12 & 5 \\
$\mathrm{M}_{1}$ & 0 & 0 \\
\hline
\end{tabular}

ISO: infección del sitio operatorio 
la pared incidida y hace relación al espacio anatómico manipulado durante el acto operatorio ${ }^{17-19}$.

En el caso específico del periné, después de la resección abdomino-perineal, se puede encontrar infección del sitio operatorio: superficial, que se manifiesta como enrojecimiento de la herida (celulitis); profunda, que compromete los planos subcutáneos grasos y el músculo elevador del ano, o de órgano y espacio, con colecciones en el espacio presacro, debajo del pliegue peritoneal que usualmente queda cerrado, separando este espacio de la cavidad abdominal.

La evaluación de pacientes con cáncer colorrectal sometidos a resección abdómino-perineal más colocación de malla de colágeno impregnada con gentamicina, para detectar infección del sitio operatorio y sus factores relacionados, permite extraer las siguientes conclusiones.

El uso de la malla con gentamicina parece tener un efecto positivo para la reducción de la infección perineal del sitio operatorio, ya que su incidencia en la muestra seleccionada fue de $41 \%$, menos que la reportada en la literatura científica, que es de 50 a $70 \%$. Asimismo, la colocación de este tipo de malla es segura, fácil, reproducible y no aumenta la morbilidad del procedimiento por rechazo local de la malla o por reacciones secundarias, como ototoxicidad y nefrotoxicidad, asociadas a la administración de aminoglucósidos.

El principal factor de riesgo para la infección perineal del sitio operatorio que se observó en este estudio fue el antecedente de neoadyuvancia, utilizada en algunos pacientes con tumores $T_{2}$ y en todos aquellos con tumores $T_{3} y_{T_{4}}$. Esto podría explicarse porque la fibrosis por radiación disminuye la penetración del antibiótico profiláctico convencional.

Otros posibles factores que podrían relacionarse con la presentación de infección del sitio operatorio, son la edad mayor de 60 años, pertenecer al sexo masculino y la localización del tumor en el recto inferior. Cabe resaltar que el riesgo por edad y sexo puede ser secundario a la distribución por sexo de la muestra y la mayor prevalencia de cáncer colorrectal en pacientes de sexo masculino. Por otra parte, la mayor frecuencia de infección del sitio operatorio en los tumores del recto inferior parece estar relacionada con el hecho de que todos los pacientes con tumores del recto inferior son sometidos a resección abdómino-perineal porque para lograr la resección oncológica, hay que sacrificar el aparato esfinteriano en la mayoría de los casos. Finalmente, no parece haber relación entre el tiempo quirúrgico y el riesgo de infección.

Teniendo en cuenta que este es un estudio observacional y descriptivo, no es posible sacar conclusiones definitivas, así como tampoco es posible extrapolar los resultados obtenidos a la población general. En el futuro, se debe realizar un estudio de mayor poder estadístico, cuyos resultados permitan recomendar la malla de colágeno con gentamicina en el protocolo institucional de manejo.

\title{
Reduction of perineal infection following abdominoperineal resection with the use of gentamicin-collagen fleece
}

\begin{abstract}
Background: Abdominal-perineal resection in patients with rectal cancer undergoing preoperative radiotherapy exhibit as a frequent complication the perineal infection of the operative site, manifested as cellulitis to perineal collections and wound dehiscence, which significantly increases hospitalization time.

Reducing the incidence of operative site infection helps to diminish perioperative morbidity and mortality. The use of local antibiotics in the surgical wound has been reported in the literature as a protective factor with promising results to avoid this complication
\end{abstract}

Methods: This is a case series report to study the outcome of patients undergoing abdominoperineal resection with the implantation of a gentamicin-collagen fleece in the perineal closure. Patients included were treated by 
the Department of Coloproctology of the Hospital Universitario San Ignacio (HUSI), Bogotá, Colombia. The main purpose of the study was to describe the institutional experience with the utilization of the gentamicin-collagen fleece to reduce the risk of surgical site infection.

Results: Twelve patients were included. Surgical site infection incidence was $41 \%$ when the gentamicin-collagen fleece was used. Nine patients had received neoadyuvant therapy and three were taken to surgery without previous neoadyuvant therapy. Five patients developed surgical site infection, superficial surgical site infection was present in three and organ/space surgical site infection developed in two cases requiring surgical drainage. There was no evidence of adverse reactions with the use of the gentamicin-collagen fleece.

Conclusions: The results show a positive effect of the gentamicin-collagen fleece in reducing the frequency of surgical site infection. Patients who received neoadyuvant therapy had a higher risk of surgical site infection. There is no need to modify the surgical technique and there is no increase in the procedures' morbidity with the use of the gentamicin-collagen fleece. Even though the results indicate a possible reduction in the surgical site infection incidence, further studies are required to obtain statistically significant conclusions.

Key words: Rectal neoplasms; prostheses and implants; collagen; anti-bacterial agents; postoperative complications; surgical wound infection.

\section{Referencias}

1. Nissan A, Guillem JG, Paty PB, Douglas Wong W, Minsky B, Saltz L, Cohen AM. Abdominoperineal resection for rectal cancer at a specialty center. Dis Colon Rectum. 2001;44:27-35.

2. Bullard KM, Trudel JL, Baxter NN, Rothenberger DA. Primary perineal wound closure after preoperative radiotherapy and abdominoperineal resection has a high incidence of wound failure. Dis Colon Rectum. 2005;48:438-43.

3. Rullier E, Laurent C, Garrelon JL, Michel P, Saric J, Parneix M. Risk factors for anastomotic leakage after resection of rectal cancer. Br J Surg. 1998;85:355-8.

4. Petrelli NJ, Nagel S, Rodríguez-Bigas M, Piedmonte M, Herrera L. Morbidity and mortality following abdominoperineal resection for rectal adenocarcinoma. Am Surg. 1993;59:400-4.

5. Berard F, Gandon J. National Academy of Sciences-National Research Council, Division of Medical Sciences. Postoperative wound infections: The influence of ultraviolet irradiation of the operating room and various other factors. Ann Surg 1964;160(Supl.2):1-192.

6. Baum ML, Anish DS, Chalmers TC, Sacks HS, Smith H Jr, Fagerstrom RM. A survey of clinical trials of antibiotic prophylaxis in colon surgery: Evidence against further use of no-treatment controls. N Engl J Med. 1981;305:353-6.

7. Hasleton PS, Carr N, Schofield PF. Vascular changes in radiation bowel disease. Histopathology. 1985;9:517-34.

8. Sachweh D. The local application of antibiotics in soft-tissue surgery. Reconstr Surg Traumatol. 1988;20:63-72.

9. Rutten HJ, Nijhuis PH. Prevention of wound infection in elective colorectal surgery by local application of a gentamicincontaining collagen sponge. Eur J Surg. 1997;578:31-5.

10. Gruessner U, Clemens M, Pahlplatz PV, Sperling P, Rosen HR, Septocoll Study Group. Improvement of perineal wound healing by local administration of gentamicin-impregnated collagen fleeces after abdominoperineal excision of rectal cancer. Am J Surg. 2001;182:502-9.

11. Friess W. Drug delivery systems based on collagen. Aachen: Shaker Verlag; 2000.

12. Mehta S, Humphrey JS, Dchenkmann DI, Seaber AV, Vail TP. Gentamicin distribution from collagen carrier. J Orthop. 1996;14:749-54.

13. Taylor EW. Surgical infection: Current concerns. Eur J Surg. 1997;163(S578):5-9.

14. Adam IJ, Mohamdee MO, Martin IG, Scott N, Finan PJ, Johnston $\mathrm{D}$, et al. Role of circumferential margin involvement in the local recurrence of rectal cancer. Lancet. 1994;344:707-11.

15. Fry D. The prevention of surgical site infection in elective colon surgery. Scientifica (Cairo). 2013;2013:896297.

16. Santalla A, López-Criado MS, Ruiz MD, Fernández-Parra J, Gallo JL, Montoya F. Infección de la herida quirúrgica. Prevención y tratamiento. Surgical site infection. Prevention and treatment. Clin Invest Ginecol Obstet 2007;34:189-96

17. Serrano-Arenas M. Infección de la herida quirúrgica. Rev Colomb Cir. 1998;13:150-7.

18. Mangram A, Horan T, Pearson M, Silver L, Jarvis W. The hospital infection control practices advisory committee. Guideline for prevention of surgical site infection, 1999. Infect Control Hosp Epidemiol. 1999;20:247-8.

19. Arias C, Quintero G, Vanegas B, Rico C, Patiño J. Surveillance of surgical site infections: Decade of experience at a Colombian tertiary care center. World J Surg. 2003;27:529-33.

Correspondencia: Cristina Isabel Martínez correo electrónico: cristinamartinezcx@gmail.com Medellín, Colombia 